\title{
Using Computational Plant Science Tools to Investigate Morphological Aspects of Compensatory Growth
}

\author{
David Thornby, Michael Renton, and Jim Hanan \\ Centre for Plant Architecture Informatics, University of Queensland, Brisbane, QLD \\ \{dft, mrenton, jim\}@cpai.uq.edu.au \\ http://www.cpai.uq.edu.au
}

\begin{abstract}
Models of cotton plant architecture expressing several physiological hypotheses about plant resource use and responses to damage are incorporated in the traditional research cycle to investigate the phenomena of compensation for defoliation. Two separate approaches to modelling the uptake and allocation of carbon are used: a detailed bottom-up physiology model expressing ideas about local control, and a top-down, canonical approach where qualitative knowledge about plant responses to defoliation are modelled as flows between plant physiological compartments. The two models provide contrasting methods for developing explanations for the underlying pattern of responses observed in the plants.
\end{abstract}

\section{Introduction}

The basic tool in a computational plant scientist's tool kit for studying biological effects related to plant architecture is a system for expressing models of plant development on a component-by-component basis. L-systems [1]allow such models to be expressed. Well-established techniques for developing models from relationships observed in real plants [2] are employed. These models can be used to express multiple hypotheses about plant behaviours, producing visual simulations and data output that capture emergent properties of the interactions among a number of hypotheses. We use this approach to investigate aspects of plant resource use and damage responses. This paper will show how the addition of a simulation phase to the normal investigative protocol of hypothesis and experimental phases aided in developing our understanding of responses to defoliation in cotton. We also aim to illustrate some of the differences between canonical and explicit approaches to modelling plant physiology, highlighting strengths and weaknesses of each.

Background It is well established that cotton crops have a high ability to compensate for significant levels of damage of various forms [3, 4], including defoliation $[5,6]$, particularly when damage occurs early in the season. That is, cotton crops are able to produce 'normal' yields (seed mass, lint mass and quality) following the removal of quite significant quantities of leaf tissue. A 
considerable body of work quantifying crop-wide yield compensation under a variety of conditions already exists. However, the morphogenetic mechanisms of the compensatory response and the architectural variables of the response (for example, leaf area or stem length changes) are not well understood.

We used L-systems $[1,7]$ to develop our models of cotton growth and damage responses. Full text of the model code and visualisations are available at www.cpai.uq.edu.au.

\section{Initial Model}

An initial model of cotton seedlings was developed to aid in the generation of morphological hypotheses concerning main stem extension in cotton and its response to defoliation. This initial model links hypothetical physiological mechanisms with morphological outcomes. Leaves near the top of the plant tend to supply their photosynthetic output upwards, towards the apex [8]. Therefore, the central hypothesis of the model is that leaf photosynthetic output near the top of the plant makes a significant contribution to the development of new metamers, defined here as an internode, leaf, and axillary bud, by the apex (Hypothesis 1). Consequently, any significant defoliation near the top of the plant would affect the period of time between the appearance of successive metamers of the main stem (the plastochron), slowing development (Hypothesis 2). It has been shown that plants may respond to defoliation by increasing photosynthetic output from remaining or newly produced leaf tissues for a period of time $[9,10]$ (Hypothesis 3). The expansion of new leaves and internodes may also at some point be limited by reduced availability of photosynthate (Hypothesis 4) [9, 10]. Apical development In the model, apical development is controlled by a resource pool. The upper leaves contribute to the pool, while apical development consumes the resources, once the pool has passed a threshold value. This process may occur multiple times in one time step (a day), to allow for development of more than one set of parts. As new parts are produced, they are assigned a number identifying their node of attachment on the main stem.

Mechanism for accumulating photosynthate to fuel apex development The amount of resources each leaf adds to the apex resource pool is a function of the size of each leaf, its proximity to the apex, and its current rate of photosynthesis. Thus, each leaf within a user-defined distance from the apex contributes resources to the pool. The apex checks its resource pool after all the relevant leaves have contributed resources for the day.

Mechanisms for modelling the effects of defoliation In the defoliated model, leaf area is removed in the appropriate locations and times, and a signal from the wound site causes a rate modifier to be increased. After a user-defined time period, it is reset. The rate modifying variable alters the rate of photosynthesis and of expansion of new leaves and internodes, ultimately changing their fully expanded size.

Initial model output Morphological predictions were made from the initial model for leaf area, plant stem length, and internode number (Fig 1). Consis- 
tent differences between the two plants are apparent, in particular, for leaf area per plant. The difference becomes apparent soon after defoliation, and a reduction in rate of increase in leaf area around steps 38-45 is primarily responsible for the significant difference throughout the rest of the simulation. Significantly, the increase in photosynthetic output following defoliation results in the plant developing new metamers more rapidly for a period of time (from defoliation to about step 32). Then, as the photosynthetic advantage is lost, the defoliated plant's plastochron increases compared to the control plant, with the result that although the plastochrons are later identical, the defoliated plant consistently lags behind. These observations led us to derive new morphological and morphogenetic hypotheses:

Hypothesis 5: Defoliated plants will manifest reduced leaf area at the end of the experiment compared to control plants.

Hypothesis 6: Defoliated plants will manifest reduced main stem length at the end of the experiment compared to control plants.

Hypothesis 7: That there would be a period where the main stem plastochron of defoliated plants would be shorter, then longer, than for undefoliated plants, resulting in fewer main stem nodes on defoliated plants.

Hypothesis 8: That leaves and internodes produced soon after defoliation would be smaller than the corresponding components on undefoliated plants. An initial experiment was designed to test these morphological hypotheses.

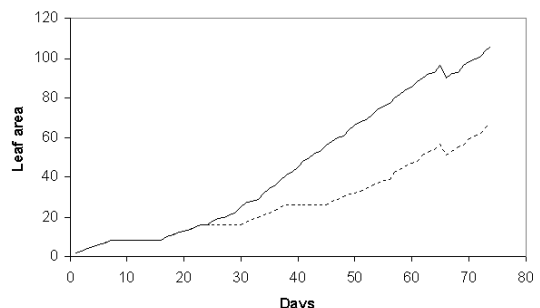

a) b)

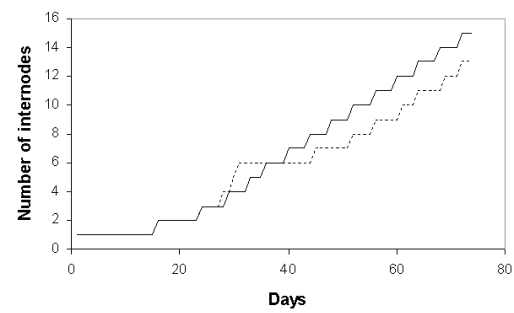

Fig. 1. Initial model predictions: a) Leaf area per day per plant. b) Number of main stem internodes per plant. Solid line: control plant; dotted line: defoliated plant

\section{Experiment}

Materials and Methods The experimental design consisted of a control group and three treatments: the removal of one of the cotyledons, the first true leaf, or both a cotyledon and the first true leaf. Twelve plants were subjected to each treatment. Gossypium hirsutum 'Sicala 40' was chosen due to its upright growth habit, reducing 'lodging' of the plants as they mature, (L. Wilson, pers. comm.) and because it shows architectural characters broadly typical of normalleaf cotton varieties. 
The plants were grown in a glasshouse, providing approximately $80 \%$ of full outdoor irradiance and photoperiod of approximately 15 hours day/9 hours night. Relative humidity inside the glasshouse was maintained at approximately $60 \%$. The plants were sown into plastic containers (20 cm diameter, $20 \mathrm{~cm}$ deep) containing a medium made up of composted pine bark (60\%), coarse sand (20\%) and peat $(20 \%)$.

The plants were allowed to grow until the majority of first true leaves were fully expanded, at which point the vigorously growing, straight-stemmed plants were assigned randomly into treatments. Defoliation was of whole laminae, using secateurs, and petioles were left intact. Plant architectures were measured using a SAC Freepoint G12 sonic digitiser and Floradig software.

Results There were no significant differences in main stem node number between treatments, either during (Fig 2) or at the end of the experiment (Fig 3). Leaf area (Fig 3 b) was not significantly affected by any of the treatments applied, thus showing full compensation.

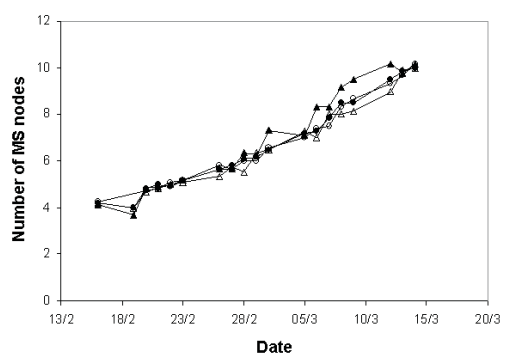

Fig. 2. Mean number of main stem internodes present per day during the experiment. - control plants; o remove one cotyledon; $\triangle$ remove first true leaf; $\triangle$ remove both

a)

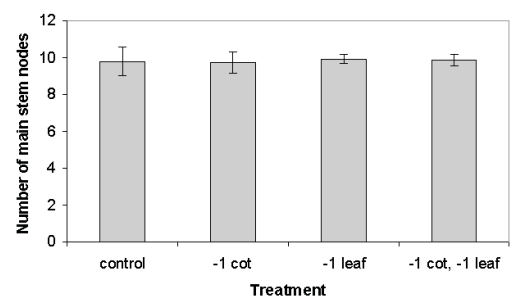

b)

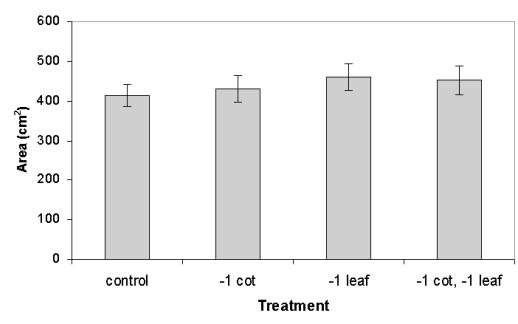

Fig. 3. Plant growth at the end of the experiment: a) Mean number of main stem internodes per plant. b) Mean lamina area. Error bars are one standard error 
Discussion Several aspects of the cotton plants' growth and development did not fit with hypotheses generated using the initial model. Firstly, main stem plastochron was not perturbed by defoliation, at least at the levels tested in experiment one. It follows that main stem meristem activity is either not carbohydratelimited, or not limited at the levels tested in experiment one. Secondly, the size of new leaves and internodes was not simply reduced by defoliation, but increased by up to $20 \%$ for a short period of time following the removal of true leaves. Conversely, the removal of a cotyledon at the seedling stage decreased the size of newly produced leaves and internodes for a short period. This may have been due to reduction in supply of some nutrient or nutrients from the cotyledons, or a change in the production of some regulatory signalling compound. Leaves and internodes at positions 3 and 4 were not fully expanded at the time of defoliation, therefore should have exhibited a size difference between treatments once fully expanded. The first metamers with different sizes were not measured as present at the time of defoliation, except as primordia. Leaves already in the rapid expansion phase were not affected by the signalling and/or resource changes caused by the defoliation treatments.

Thirdly, for leaf area and main stem length, the treated plants were able to compensate fully - that is, no significant differences were found between treatments for these morphological characters. Therefore, main stem development and growth rates (and thus absolute growth rate in pre-flowering, unbranched seedlings) are not a function of the amount of leaf area left on the plants after defoliation. Where the initial model predicted that defoliated plants would be unable to carry as large a reproductive biomass as undamaged plants [11], the data from the experiment suggests otherwise.

\section{Improved Physiologically Explicit Model}

To capture the results of the initial experiment, either quantitative (parameter changes to existing mechanisms) or qualitative (changes of mechanism) changes must be made. Reasonable quantitative changes did not allow the model to simulate the responses observed in the data; in order to have the model compensate fully, the rate modifier needed to be adjusted upwards to above 2, and to last for several weeks - well beyond any level likely to be observed in real plants. Within the experiment's limits, the main stem appears to develop obligately (according to a predetermined plan), not facultatively (according to current resource levels). This appears to be a significant contributor to the ability of these plants to compensate for defoliation in the tested indices such as leaf area (Fig $3 \mathrm{~b}$ ). Therefore, we made qualitative changes to the physiological hypotheses underlying the model, by replacing the explicit mechanisms for plastochron dependence on levels of substrate carbon (Hypotheses 1-3) by the hypothesis that the plastochron is determined only by accumulated day-degrees [7].

Changes made to the initial model The value of the day-degree requirement of each successive main stem plastochron is determined using values appropriate to the current length of the main stem, in node numbers. While carbohydrate 
availability was removed from the decision-making process concerning main stem meristem activity, carbohydrate production, transport and utilisation are used as important drivers of the successful development of plant parts in the new model. Local levels of available carbohydrate are stored for each main stem node position. Substrate carbohydrate is produced in the leaves according to grams per square centimetre values for cotton leaves given by Constable in his examinations of transport of radioactive-labelled carbon in cotton plants [12, 13]. Leaf productivity increases to a peak at about 7 days after production, and then declines with age [12].

Carbohydrate is transported upwards and downwards between main stem node positions, with values transported in each direction determined by each node's distance from the apex.

Future potential maximum sizes of parts are determined in the primordial phase. When the apex develops, it produces a primordium, rather than a fully differentiated set of plant organs (internode, bud, and leaf). The primordium carries a parameter that affects the size of the leaf and internode that it will eventually become. For each day that perturbed conditions exist in the plant, the potential maximum size of future metamers is adjusted.

Successful expansion of new parts (leaf, internode, flower bud, boll) to full size depends on local availability of substrate carbohydrate. Reproductive structures have the highest priority over local supplies of substrate carbohydrate and will be abscised if insufficient carbohydrate is available over a length of time.

Output from the improved explicit model By changing the model in the ways specified above, the plant is able to compensate more or less fully for defoliation applied in the simulation. The results for leaf area (Fig 4) show high levels of compensation for defoliation. Number of main stem nodes over time is exactly the same for each treatment.

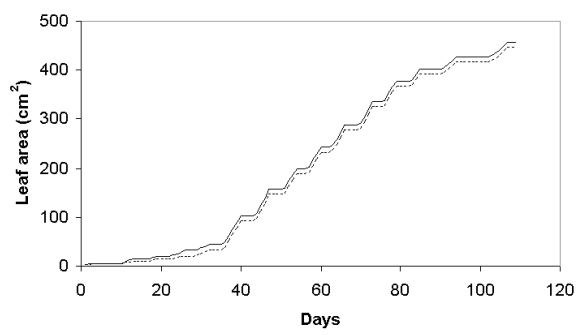

Fig. 4. Leaf area per plant, predicted by improved physiologically explicit model. Solid line: undefoliated; dotted line: two leaves removed at start.

Discussion Some aspects of the initial physiology model remain: notably, the effect of defoliation on the rate of photosynthesis. This illustrates one of the shortcomings of the morphological approach to experimentation: that is, we did 
not measure the rate of photosynthesis and so could not evaluate any changes to photosynthetic output following defoliation. Therefore, while our morphological data did not dispute the theory of an increased photosynthetic rate, neither do we have direct information on the phenomenon. The physiologically explicit model leads us to believe that such a mechanism is at least possible, and indicates that it would be a partial explanation for the level of compensation found in the experimental results. We therefore retained it as one of the physiological hypotheses present in the model. However, we were not able to improve that aspect of our model by, for example, more accurate (data-supported) estimation of rate parameters. The parameters for changing the value of the rate modifying variable, and for its effects on the model plant's behaviour, remain fitted by hand.

There are two alternatives for improving our ability to produce numerically accurate simulations of the interactions between cotton's physiology and morphogenesis: either improve the physiologically explicit model by gathering more directly physiological data, or model the physiology at a more abstract level.

\section{The Canonical Model}

Where we encountered a need for missing pieces of information on aspects of cotton's physiological processes, the construction of a physiologically explicit model became difficult and less rigorous than we would have liked. In order to address this, we decided to model the physiology at a more abstract level [14]. The canonical approach [15] is particularly useful for modelling the qualitative and quantitative behaviour of physiological systems where detailed knowledge about the system is unavailable, when gaps exist in detailed knowledge, or when a detailed physiological mechanism is not needed. Similar canonical models have been effective in modelling birch tree responses to damage [15], forest stand dynamics [16, 17] and dry matter partitioning in trees [18].

Construction First we decided which variables would be important for our model and assigned each one a 'compartment' $x$. We then used a series of differential equations to represent flows of resources between compartments. The equations are of a simple, robust power law form: flux $=k x^{h}$, where $k$ and $h$ are fitted constants.

In our case, the compartments and flow equations represent different aspects of the carbohydrate physiology system in cotton: leaf biomass, stem biomass and storage (Fig 5). We also added a 'response' compartment and a flow for 'lost' carbon resource (representing carbon that is actually lost from the system as well as that which becomes fixed in structural components other than leaf and stem). Resources flow into the system and between the compartments, altering the state variables corresponding to each compartment.

Various aspects of physiology are simplified or implied. For example, the existence and characteristics of the storage pool are only qualitatively known, and the exact character of the response compartment is unknown. The structure 
a)

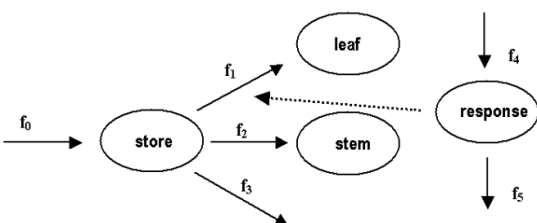

loss

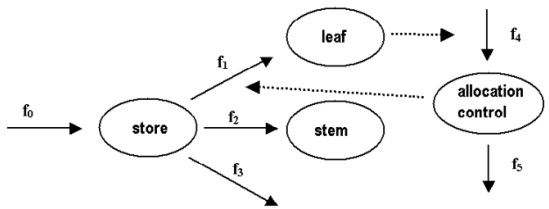

b)

loss

Fig. 5. Flow diagrams for the canonical model. The ellipses represent compartments. The solid arrows represent flows between compartments. The dotted arrow represents the influence that a compartment has over a flow. a) The 'presence' version. b) The 'absence' version

of the canonical physiology model is based on qualitative assumptions about the system.

In the case of our model, the primary aim was to demonstrate the process of compensation, to assist in constructing hypotheses - and therefore further experiments - about compensation, and to simulate the effects of presuming these hypotheses to be true. Clearly, in order for the cotton plant to compensate for damage in a mathematical model, there must be a response - a mechanism for changing the behaviour of the system so that it returns to the same state as an undefoliated system. The basis of our model's response mechanism is the adjustment of allocation patterns away from storage towards leaf and stem growth.

This response was modelled in two different ways. Firstly, in the 'presence' version, a defoliation event stimulates the production of a response, which could be a cascade of compounds in real plants. The response compartment alters the system's allocation strategy while it is present, and decays after its initial stimulation. Secondly, in the 'absence' version, an 'allocation control' compound is produced consistently at a rate proportional to the leaf biomass pool and decays at a constant rate; upon the removal of some portion of the leaf mass (i.e. defoliation), the size of the 'allocation control' pool drops rapidly, causing the response variable to increase in inverse proportion. The change in the response variable alters the allocation pattern, again away from storage towards leaf and stem growth.

The values of the constants for these equations were determined by evolutionary optimisation algorithms; the model was fitted to a subset of the data from the experiment. The model was solved using the Euler method for differential equations.

The model displays near-complete compensation for leaf biomass (Fig 6), which fits with the experimental data.

\section{Conclusions}

Expressing our hypotheses in a dynamic model has enabled us to investigate their interactions in a concrete manner. The model that assumed increased leaf production rate was tested against an experimental data set and required adjust- 

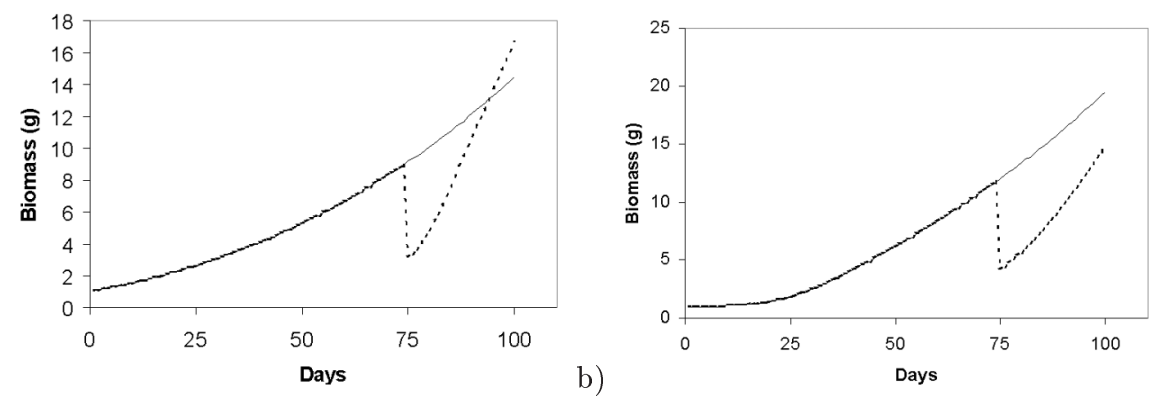

Fig. 6. Leaf biomass per plant, predicted by the canonical model. Solid line: undefoliated; dotted line: defoliated $65 \%$ at day 75 . a) presence version; b) absence version

ment. The new model incorporated obligate development of the main stem, as this appears to be a significant contributor to the ability of these plants to compensate for defoliation in the tested indices such as leaf area and plant height. Uninterrupted development of new metamers on the main stem allows for replacement of lost leaf area without depending significantly on the remaining leaf area after defoliation, therefore the model is able to compensate for the lost leaf tissue. This physiologically explicit model is well suited to testing specific aspects of physiology. However, it is very sensitive to incomplete or incorrect knowledge (whether qualitative information or quantitative data).

The alternative, canonical model is particularly effective at demonstrating compensation for defoliation. Unlike the physiologically explicit model, it simulates compensation using a whole-plant controlling mechanism - the adjustment of resource allocation away from storage and into vegetative parts. Because it is mechanistic at a global level, it can be parameterised to fit a set of observations relatively easily. While not giving insights into interacting physiological hypotheses at a local level, it does allow us to investigate other aspects of the system. While both 'absence' and 'presence' models achieve compensation, only the 'presence' model can simulate over-compensation. If experimental results showed overcompensation, this would lend support to the 'presence' mechanism at the expense of the 'absence' mechanism.

We intend to investigate the issue further by producing a hybrid model: one that uses a canonical model to determine physiological parameters, which would then be passed to an architectural model for allocation to structure. The level of interaction between the two models may be flexible; would probably be the major part of the problem of getting the two together. It is our hope that the structural model will provide a method for improving the canonical model's realism, while the canonical model should help avoid the problems of inconsistent or missing data we have observed in constructing the structural model with explicit physiology. 


\section{References}

1. Prusinkiewicz, P.: A look at the visual modelling of plants using L-systems. Agronomie: agriculture and environment 19, 211-224 (1999)

2. Hanan, J.S. and Room, P.M.: Practical aspects of virtual plant research, In Michaelewicz, M.T. (ed), Plants to ecosystems. Advances in computational life sciences. CSIRO, Melbourne, 28-44 (1997)

3. Sadras, V.O. and Fitt, G.P.: Resistance to insect herbivory of cotton lines: quantification of recovery capacity after damage. Field crops research 52, 127-134 (1997)

4. Mascarenhas, V.J., Cook, D., Leonard, B.R., Burris, E. and Graves, J.B.: Late season beet armyworm (Lepitoptera: Noctuidae) infestations on cotton: defoliation, fruit damage, and yield loss. Florida entomologist, 82(2), 218-229 (1999)

5. Longer, D.E. and Oosterhuis, D.M.: Cotton regrowth and recovery from early season leaf loss. Environmental and experimental botany, 41, 67-73 (1999)

6. Longer, D.E., Oosterhuis, D.M., and Withrow, M.: Cotton seedling recovery from partial and complete defoliation. Arkansas Farm Research, 42, 10-11 (1993)

7. Hanan, J.S.: Virtual plants - integrating architectural and physiological models. Environmental modelling and software, 12(1), 35-42 (1997)

8. Ashley, D.A.: ${ }^{14}$ C-labelled photosynthate translocation and utilization in cotton plants. Crop science 12, 69-74 (1972)

9. Meyer, G.A.: Pattern of defoliation and its effect on photosynthesis and growth of goldenrod. Functional ecology 12, 270-279 (1998)

10. Verkaar, H.J.: Are defoliators beneficial for their host plants in terrestrial ecosystems? - a review. Acta Botanica Neerlands 37(2), 137-152 (1988)

11. Ashley, D.A., Doss, B.D. and Bennett, O.L.: Relation of cotton leaf area to plant growth and fruiting. Agronomy journal, 61-64 (1964)

12. Constable, G.A.: Section 7: Distribution of ${ }^{14} \mathrm{C}$ photosynthate in cotton, in Carbon fixation and distribution in cotton: implications of single-leaf measurements to plant performance, Ph.D. Dissertation, 82-122 (1981)

13. Constable, G.A. and Rawson, H.M.: Effect of leaf position, expansion and age on photosynthesis, transpiration and water use efficiency of cotton. Australian journal of plant physiology, vol 7, 89-100 (1980)

14. Renton, M., Hanan, J. and Kaitaniemi, P.: The inside story: including physiology in structural plant models. Proceedings of the international conference on computer graphics and interactive techniques in Australiasia and South-East Asia (GRAPHITE2003), 95-102 (2003)

15. Kaitaniemi, P.:A canonical model of tree resource allocation after defoliation and bud consumption. Ecological modelling 129, 259-272 (2000)

16. Martin, P.G.:The use of canonical S-system modelling for condensation of complex dynamic models. Ecological modelling 103, 43-70 (1997)

17. Torres, N.V.: S-system modelling approach to ecosystem: application to a study of magnesium flow in a tropical forest. Ecological modelling 89, 109-120 (1996)

18. Voit, E.O. and Sands, P.J.: Modeling forest growth II - biomass partitioning in Scots pine. Ecological modelling 86, 73-89 (1996) 